Research Article

\title{
Smooth Muscle Progenitor Cells Preserve the Erectile Function by Reducing Corporal Smooth Muscle Cell Apoptosis after Bilateral Cavernous Nerve Crush Injury in Rats
}

\author{
Yi-No Wu, ${ }^{1}$ Kuo-Chiang Chen, ${ }^{1,2}$ Chun-Hou Liao $\left(\mathbb{D},{ }^{1,3}\right.$ Chien-Liang Liu, ${ }^{4}$ \\ and Han-Sun Chiang $\mathbb{D}^{3,5,6}$ \\ ${ }^{1}$ School of Medicine, Fu Jen Catholic University, New Taipei City, Taiwan \\ ${ }^{2}$ Department of Urology, Cathay General Hospital, Taipei, Taiwan \\ ${ }^{3}$ Division of Urology, Department of Surgery, Cardinal Tien Hospital, New Taipei City, Taiwan \\ ${ }^{4}$ Division of Urology, Department of Surgery, Chi Mei Hospital, Tainan City, Taiwan \\ ${ }^{5}$ Graduate Institute of Biomedical and Pharmaceutical Science, Fu Jen Catholic University, New Taipei City, Taiwan \\ ${ }^{6}$ Department of Urology, Fu Jen Catholic University Hospital, New Taipei City, Taiwan \\ Correspondence should be addressed to Han-Sun Chiang; yino58@hotmail.com
}

Received 12 November 2018; Revised 7 May 2019; Accepted 22 October 2019; Published 16 November 2019

Academic Editor: Aijun Wang

Copyright (c) 2019 Yi-No Wu et al. This is an open access article distributed under the Creative Commons Attribution License, which permits unrestricted use, distribution, and reproduction in any medium, provided the original work is properly cited.

\begin{abstract}
Radical prostatectomy causes erectile dysfunction (ED) and irreversible morphologic changes, including induction of endothelial and smooth muscle cell (SMC) apoptosis in the corpus cavernosum (CC). The injection of smooth muscle progenitor cells (SPCs) thickens the vascular intima and has demonstrated therapeutic benefit in cardiovascular disease animal. Herein, we investigated the effect of SPCs on the recovery of erectile function (EF) in rat models with bilateral cavernous nerve (CN) injury. Twenty-four male Sprague-Dawley rats were randomized into sham, vehicle only, or SPC treatment groups. Rats in the SPC treatment and vehicle groups were subjected to bilateral CN injury before intracavernosal injection. Intracavernosal injections of SPCs increased all EF parameters at day 28 after injury and simultaneously reduced apoptosis of the SMCs. Ultrastructural analysis revealed that SPCs maintained the integrity of the CC by preserving the structure of the adherens junctions. Tracking transplanted SPCs labeled with EdU showed that transplanted SPCs remained in the CC 28 days after treatment. Intracavernosal SPC injection restored EF after bilateral CN injury by reducing SMC apoptosis, which favored the maintenance of the structure of adherens junctions and regulated the stability of corporal vessels. These findings demonstrate the therapeutic potential of SPCs for treating ED in humans.
\end{abstract}

\section{Background}

In urology, postprostatectomy erectile dysfunction (ED) complication occurring after radical prostatectomy (RP) for earlier prostate cancer is a topic of most concern. Nerve injury may also be the main cause of erectile dysfunction after surgery $[1,2]$. Epidemiology of ED after RP range is from $60 \%$ to $70 \%$; even when nerve-sparing techniques are applied, ED rates range is still high from $30 \%$ to $87 \%$ [3]. This type of ED is most likely due to a multifactorial process, including intraoperative neurogenic and vasculogenic injury. For many years, ED after radical surgery was overcome by the implantation of a penile prosthesis [4]. Several treatment approaches are currently available for clinical treatment after radical prostatectomy. Pharmacotherapeutic options include oral treatment (phosphodiesterase 5 (PDE5) inhibitor) and intracavernosal injections (PGE-1). The PDE5 inhibitors showed benefit only in $54 \%$ of ED patients in one study [5]. Moreover, PDE5 inhibitors cannot improve the unassisted erectile function (EF) with RP [6]. There is still no progress in terms of effective agents for ED. Our previous study was mainly to find the advance methods to protect the cavernous nerve because a neurogenic component likely plays an important role in the pathogenesis of the ED [7-9]. However, in our recent study, we found that $\mathrm{CN}$ injury induces the most severe ED and nerve damage, which is 
followed by partial spontaneous recovery of EF and regeneration of the $\mathrm{CN}$ at day 28 after injury. Defects in the corporal SMCs were irreversible after $\mathrm{CN}$ injury. This suggests that the protection of the corpus SMCs from apoptosis may represent a more important treatment modality than nerve protection in future studies using the $\mathrm{CN}$ crush injury model [10]. Therefore, an alternative treatment is highly desirable to develop an effective, clinical feasible medication for ED patients after RP.

Several pathophysiological theories have been proposed to explain $\mathrm{ED}$ after $\mathrm{CN}$ injury, including $\mathrm{nNOS}$-positive nerve fiber decrease, vascular damage, corporal cavernosum inflammatory, cavernosal smooth muscle hypoxia and smooth muscle apoptosis and fibrosis [11]. Some reports have stated that neuromodulatory treatments are possible options, such as use of immunophilin ligands, neurotrophins, growth factors, and stem cell therapy, to prevent the development of hypoxia-induced tissue damage and fibrosis. Currently, stem cell-based therapy has been a promising strategy for neuroprotection or tissue-protection after bilateral $\mathrm{CN}$ injury [12]. It is difficult to translate to clinical use because of adverse pharmacokinetics and because it is hard for cancer patients to use.

Bone marrow-derived progenitor cells (BMPCs), including endothelial progenitor cells (EPCs) and smooth muscle progenitor cells (SPCs), can be derived from the autologous peripheral blood, and they differentiate more easily into vascular cells during arterial remodeling than stem cells. EPCs are capable of circulating, proliferating, and differentiating into mature endothelial cells [13]. We have also previously reported that EPC treatment restored EF in a rat model of bilateral $\mathrm{CN}$ injury through recruitment of EPCs toward the dorsal artery and preservation of corporal SMCs. These findings support the therapeutic potential of progenitor cells for treating ED in humans [14]. SPCs can limit plaque development and promote changes in plaque composition toward a stable phenotype in mice [15]. SPCreleased angiopoietin-1 can facilitate stabilization of endothelial cell networks and may be responsible for tightly orchestrating the complex process of neovascularization. In addition, the proliferative ability of SPCs in vitro is greater than that of EPCs [16]. Close cooperation between endothelial cells and SMCs is important for the regulation of vessel maturation and stability.

The effects of intracavernous injections of SPCs for the treatment of ED after $\mathrm{CN}$ injury have not been explored to date. Given that SPCs can enhance the efficiency of proangiogenic cell-based therapy [17], the present study aimed to evaluate the therapeutic potential of SPCs injections into the corpora cavernosa of rats with bilateral $\mathrm{CN}$ injury.

\section{Methods}

2.1. Experimental Animals. Twenty-five 10-week-old male Sprague-Dawley rats (weight, 350-450 g) were used in this study. All animals were supplied by BioLasco Taiwan Co., Ltd. (Taipei, Taiwan), and approved by the Fu Jen Catholic University Animal Care and Use Committee (IACUC approval no.: A10425). All the animal experiment methods were conducted in accordance with the guidelines and regulation established by $\mathrm{Fu}$ Jen Catholic University, Taiwan.

2.2. Isolation and Culture of Smooth Muscle Progenitor Cells. Ten-week-old male Sprague-Dawley rats were used to isolate the SPCs from femurs. The bone marrow was diluted with phosphate-buffered saline (PBS). Mononuclear cells were obtained from the bone marrow by density gradient centrifugation (Histopaque-1083; Sigma-Aldrich, St. Louis, $\mathrm{MO}, \mathrm{USA}$ ) at $400 \times \mathrm{g}$ for 30 minutes and then at $300 \times \mathrm{g}$ for 10 minutes. The cell pellet was then suspended in endothelial basal medium-2 (EBM-2; Lonza Clonetics, Walkersville, MD, USA) supplemented with PDGF-BB (50 ng/mL). After 2-3 days, angioblast-like cells and spindle-shaped cell outgrowths were observed. SPCs were defined by cell morphology and immunofluorescence staining. To determine the potential activity of SPCs, cells were labeled with a longterm tracking dye using the EdU Cell Labeling Kit (Invitrogen) at different time points $(6,24$, and 48 hours) and concentrations $(10,50$, and $100 \mu \mathrm{M})$ and then harvested.

\subsection{Experimental Design and Surgical Procedures.} Cavernous nerve crushing animal model was established by a standardized procedure to generate a nerve injury as described previously [7-10]. The rats were randomly assigned to 3 groups ( 8 rats per group): sham, vehicle only, and SPCs-treated groups. For the surgical procedure, the rats were first anesthetized with an intraperitoneal injection of sodium pentobarbital $(50 \mathrm{mg} / \mathrm{kg})$. After a lower abdomen midline incision, major pelvic ganglions and the CNs were observed and isolated. All rats were undergoing bilateral $\mathrm{CN}$ injury except sham group. The crushing injury of the CNs was applied using a hemostat clamp (Roboz Surgical Instrument Co. Inc., Gaithersburg, USA) for 2 minutes before closing the abdomen.

2.4. Measurement of Erectile Responses. The measurement of erectile function was determined by electrical stimulation of the CNs at 4 weeks after surgery to generate erectile responses as described previously [7-10]. The CNs were isolated from prostate, and the crura of the penis were identified. A $24 \mathrm{G}$ needle containing $50 \mathrm{U} / \mathrm{mL}$ of heparin solution was inserted into the right penile crus. The MP36 pressure transducer (Biopac Systems Inc., CA, USA) was used to measure the intracavernous pressure (ICP) which was recorded by BSL 3.7.3 software. Then, the CNs were stimulated using a bipolar stainless steel electrode and the monophasic pulses were generated by a DS3 constant current stimulator (AutoMate Scientific Inc., CA, USA). The stimulus parameters included a $7.5 \mathrm{~mA}$ amplitude, a $20 \mathrm{~Hz}$ frequency, and a 0.2 -millisecond pulse width of 60-second duration. Erectile responses were expressed as intracavernous pressure (ICP). The maximal ICP, the change in ICP $(\triangle \mathrm{ICP})$, the area under the ICP curve (AUC), and the ratio of change in ICP and mean arterial pressure (MAP; 


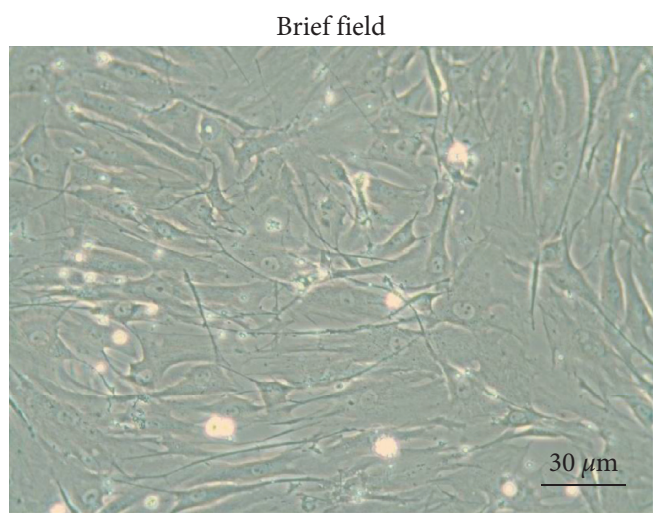

(a)

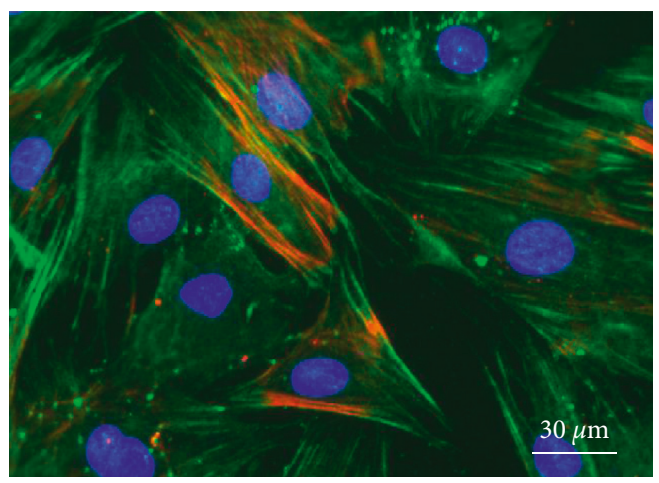

At differentiation $28 \mathrm{~d}$ $60 \%$ degree of SPC

(c)

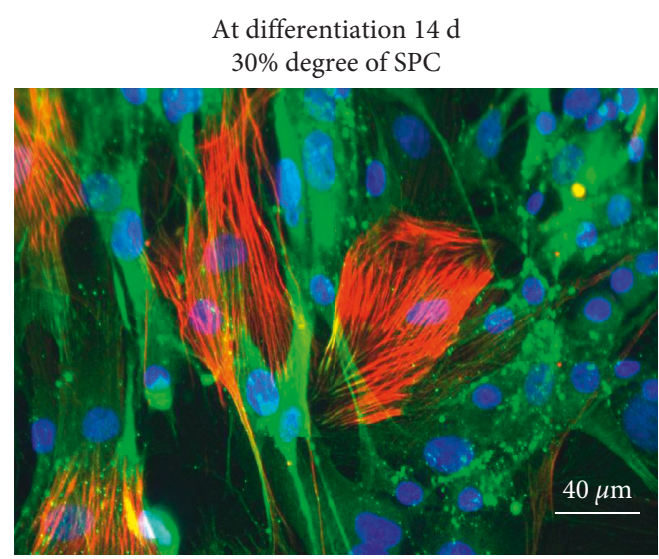

Red : $\alpha$-SMA

Green : CD 34

Blue: DAPI (b)

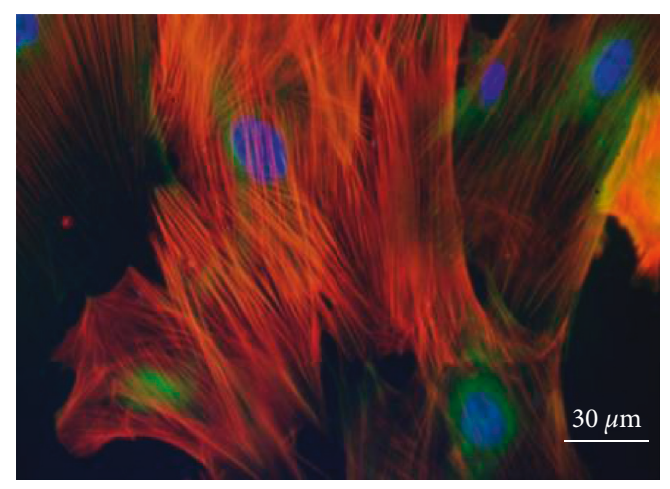

At differentiation $35 \mathrm{~d}$ $90 \%$ degree of SPC

(d)

FIGURE 1: The characteristics of smooth muscle progenitor cells. (a) Outgrowth of smooth muscle progenitor cells (SPCs) by using the phase contrast microscope. (b-d) Immunofluorescence staining of SPCs. The cells were incubated with anti- $\alpha$-SMA (red) and anti-CD34 (green) antibodies, respectively, and then complexed with Alex 594 and 488 secondary antibodies. Cell nuclei were counterstained with DAPI (blue).

$\triangle \mathrm{ICP} / \mathrm{MAP}$ ) were assessed as the parameters of erectile function.

2.5. Immunofluorescence Staining. To determine the content of nNOS-positive nerve fiber and smooth muscle content in the cavernous nerve and penile tissue, we performed double immunofluorescent labeling for cavernous related tissue with antibodies as described previously $[9,10]$. The nerve and penile tissue were fixed with formalin for 24 hours $(10 \%$ formaldehyde $\mathrm{w} / \mathrm{v}$ ) and were subsequently embedded in paraffin. In the deparaffinized procedure, the slide was put in xylene for 10 minutes and this is repeated twice for a total of 3 treatments, followed by hydration in graded alcohols. Blocking procedures were incubated for 1 hour in $10 \%$ goat serum $/ 2 \%$ bovine serum albumin $/ 0.2 \%$ Triton X-100 (Sigma-Aldrich, St. Louis, MO, USA) at room temperature. The tissues were subsequently incubated overnight at $4^{\circ} \mathrm{C}$ with the following primary antibodies: rabbit anti-nNOS (Santa Cruz Biotechnology, Santa Cruz, CA, USA), mouse anti-neuron-specific $\beta$-III tubulin, and anti- $\alpha$-smooth muscle actin (Abcam, Cambridge, UK). Then, the tissues were incubated with a $1: 400$ dilution of secondary antibody conjugated to Alexa Fluor 488 or Texas Red (Invitrogen,
Carlsbad, CA, USA) for 1 hour. For further analysis of nNOS and SMC content by fluorescence microscopy, the ratios of the area of nNOS-positive cells to the area of $\beta$-III tubulinpositive cells in nerve fibers and the $\alpha$-smooth muscle actin area of the corpus cavernosum (CC) were calculated at $400 \times$ and 50× magnifications, respectively. All computerized histomorphometric analyses were performed using ImageJ software (National Institutes of Health, Bethesda, MD, USA).

2.6. Statistical Analysis. Data were expressed as the mean\pm standard deviation. Statistical analysis for comparison of multiple treatment groups was performed with a one-way analysis of variance (ANOVA) and pairwise post hoc comparisons with the Scheffé test by using the SPSS Version 12.0 (SPSS Inc., Chicago, IL, USA) for Windows. The $p<0.05$ was considered to be statistically significant.

\section{Results}

3.1. Cultivation and Characteristic of Smooth Muscle Progenitor Cells. Approximately 30\% of the freshly isolated bone marrow mononuclear cells (BMMCs) adhered to 

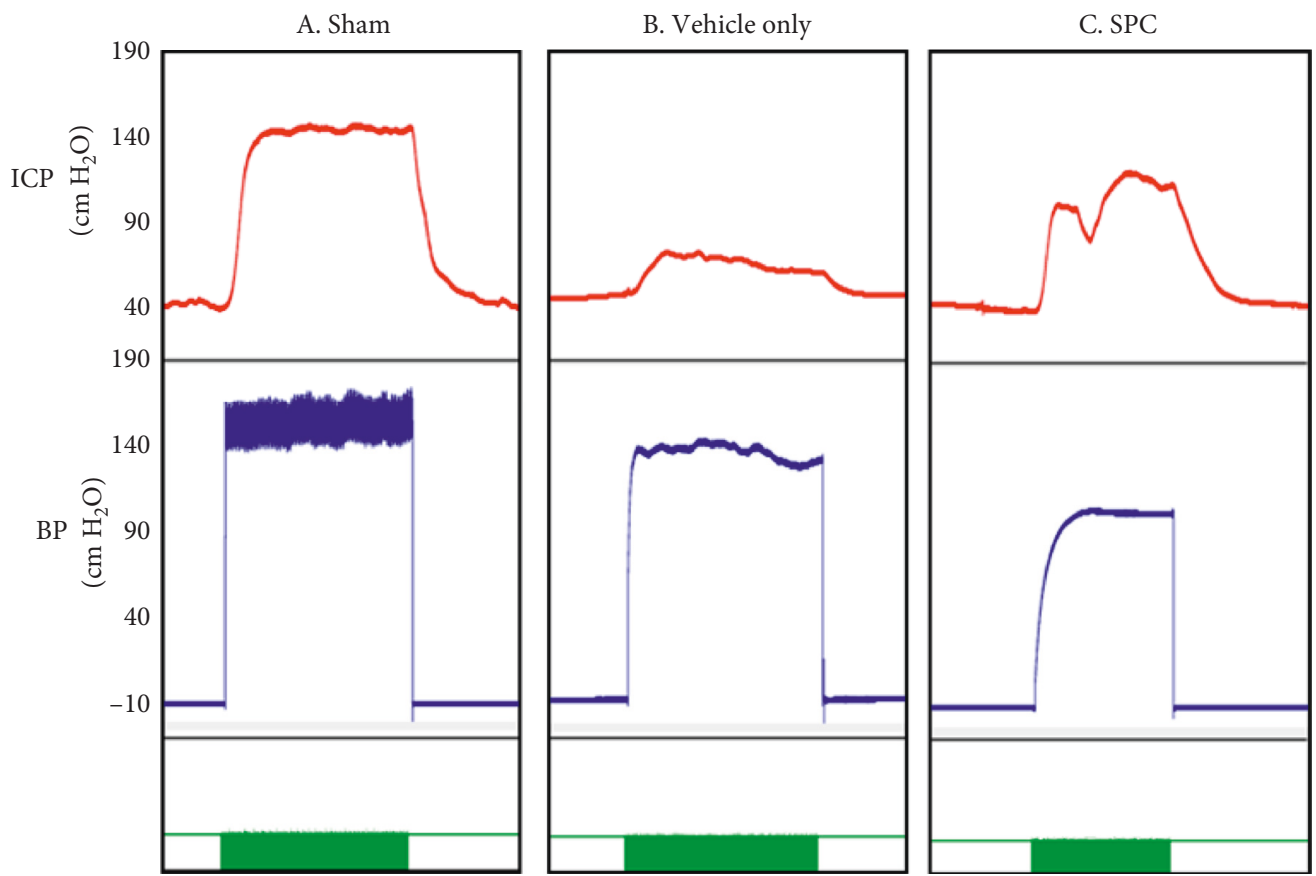

FIGURE 2: The measurement of intracavernosal pressure. Recordings of the intracavernosal pressure (ICP) and arterial blood pressure (BP) in the (A) sham, (B) vehicle only, and (C) smooth muscle progenitor cells- (SPCs-) injected mice. The $x$-axis represents time in seconds, and the green bar represents one electrical stimulus of 60 seconds. The $y$-axis represents the ICP and BP (top and bottom panels) in the experimental animals $(n=8)$.

TABLE 1: Intracavernous and mean arterial pressure measurements on cavernous nerve electrostimulation.

\begin{tabular}{lccccc}
\hline Groups & Maximum ICP $\left(\mathrm{cm} \cdot \mathrm{H}_{2} \mathrm{O}\right)$ & $\Delta \mathrm{ICP}\left(\mathrm{cm} \cdot \mathrm{H}_{2} \mathrm{O}\right)$ & AUC & $\Delta \mathrm{ICP} / \mathrm{MAP}$ & Maximum ICP/BP \\
\hline Sham & $141.72 \pm 12.91^{*}$ & $110.59 \pm 12.79^{*}$ & $4479.86 \pm 948.83^{*}$ & $0.68 \pm 0.10^{*}$ & $0.88 \pm 0.0 .12^{*}$ \\
Vehicle only & $56.19 \pm 9.01$ & $33.00 \pm 10.12$ & $1059.71 \pm 413.66$ & $0.19 \pm 0.06$ & $0.33 \pm 0.05$ \\
SPC & $127.85 \pm 20.89^{*}$ & $100.85 \pm 25.61^{*}$ & $5193.72 \pm 1585.04^{*}$ & $0.64 \pm 0.14^{*}$ & $0.81 \pm 0.12^{*}$ \\
\hline
\end{tabular}

$\mathrm{SPC}=$ smooth muscle progenitor cell; ICP $=$ intracavernous pressure; ICP $=$ Max ICP-Min ICP; MAP = mean arterial pressure; AUC $=$ area under curve. ${ }^{*} P<0.001$ versus vehicle only group.

culture plates after 3 days of culture with PDGF-BB-containing EGM-2-MV medium (Figure 1(a)). Two weeks after isolation and differentiation, $30 \%$ of outgrowth cells displayed specific morphological characteristics of SPCs as observed through fluorescence microscopy (Figure 1(b)). Thereafter, many SPC colonies were positive with CD34 and $\alpha$-SMA antigen after differentiation at 4 weeks (about $60 \%$ SPC) (Figure 1(c)) and at 5 weeks (about 90\% SPC) (Figure 1(d)).

\subsection{Smooth Muscle Progenitor Cells Treatment Restores} Erectile Function. Figure 2 presents the intracavernosal pressure (ICP) and the arterial blood pressure (BP) in the sham, vehicle only, and SPC-treated groups at 28 days after injury. The maximum ICP was significantly lower in the vehicle only treatment group compared with the sham group $\left(56.19 \pm 9.01\right.$ versus $141.72 \pm 12.91 \mathrm{~cm} \mathrm{H}_{2} \mathrm{O}$, respectively; $p<0.001$ ), which is consistent with ED. The maximum ICP in the SPC treatment group $\left(127.85 \pm 20.89 \mathrm{~cm} \mathrm{H}_{2} \mathrm{O}\right.$; $p<0.001$ ) was higher than that in the vehicle only group, but the difference between the SPC-treated and the sham groups was not significant. Similarly, other functional parameters of $\mathrm{EF}$, such as $\triangle \mathrm{ICP}$, area under curve (AUC), $\triangle \mathrm{ICP} / \mathrm{BP}$, and maximum ICP/BP, were significantly increased in the SPCtreated group (Table 1).

\subsection{Intracavernous Injection of Smooth Muscle Progenitor} Cells Can Prevent Atrophy of Corporal Smooth Muscle Cells. The nNOS-positive nerve fibers of the dorsal penile nerve were assessed by immunofluorescence staining. Figure 3(a) depicts the immunostaining of the dorsal penile nerves for nNOS and $\beta$-III tubulin to quantify their nNOS content. Quantitative analysis showed that the ratio of the area of expression of nNOS/ $\beta$-III tubulin was dramatically reduced in the vehicle only group compared with the sham group; however, there was no significant increase in the number of nNOS-positive nerve fibers after SPC treatment (Figure 3(b)). The corporal SMC content in the corpora cavernosa was evaluated by $\alpha$-smooth muscle actin staining (Figure 4(a)). The SMC content within the corpora cavernosa was significantly lower in the vehicle only group compared with the sham and the SPC treatment groups (all 

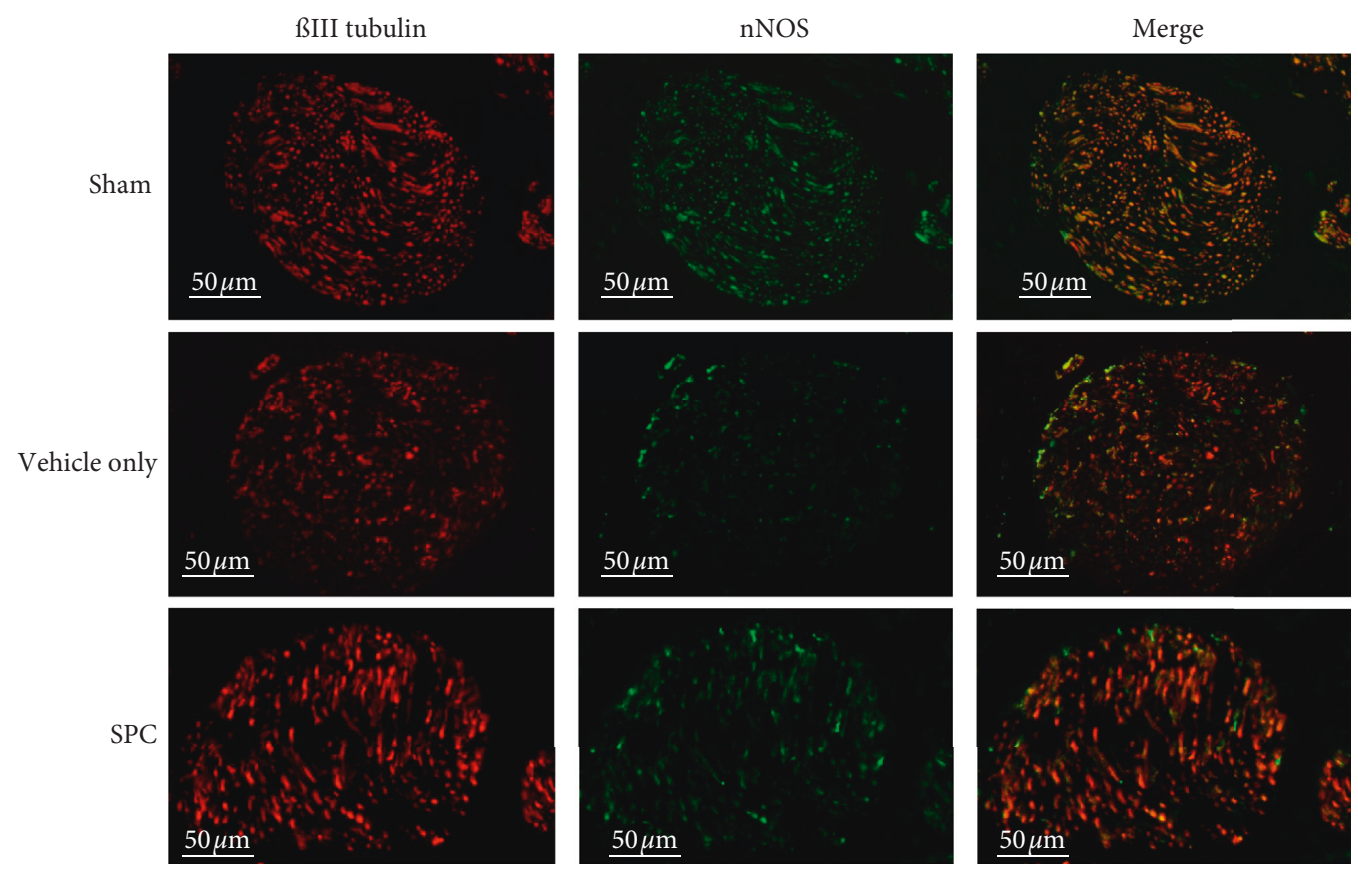

(a)

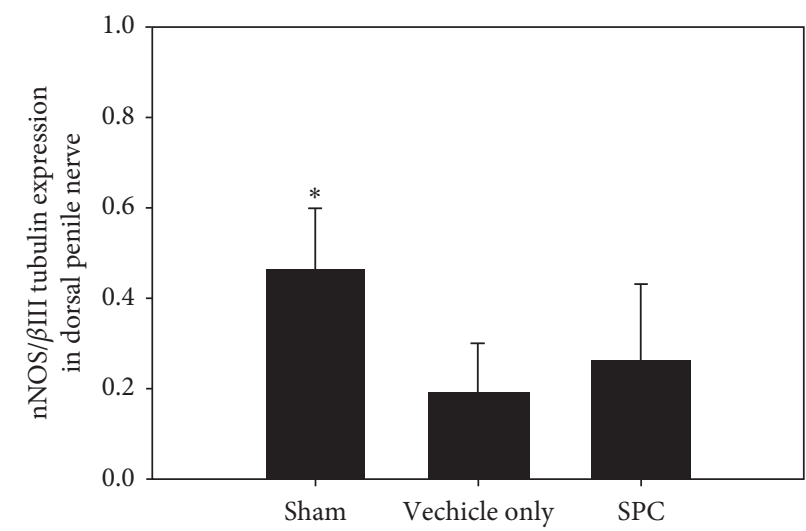

(b)

FIGURE 3: Immunofluorescence staining of nNOS in dorsal penile nerve. (a) Representative images of the dorsal penile nerve of each treatment group (original magnification: $400 \times$ ). (b) Graph showing the quantification of nNOS-positive nerve fibers in the dorsal penile nerve, calculated as the area of nNOS-positive nerve fibers/ $\beta$-III tubulin. ${ }^{*} p<0.05$ versus vehicle only group.

$p<0.05$; Figure 4(b)). These results indicate that SPC can prevent the atrophy of corporal SMCs.

Intracavernous injection of smooth muscle progenitor cells maintains the structure of adherens junctions of the smooth muscle in the corpus cavernosum.

Ultrastructural analysis of the CC tissue in the vehicle only and SPC-treated groups at 28 days after injury (Figure 5) was conducted by electron microscopy. Lowermagnification TEM images revealed healthy smooth muscle of the CC near the sinusoid in the sham groups (Figure 5(A)). Healthy smooth muscle was often located along the boundary of the endothelial cells and close to the collagen tissue. Higher-magnification micrographs suggested that the ultrastructure of the adherens junctions in the corporal tissue was intact and closely placed (Figure 5(D)). However, we observed abnormal structures and apoptosis of the SMCs in the vehicle only group (Figure 5(B)). In addition, loss of the adherens junctions in the SMC was also observed in the vehicle only group (Figure 5(E)). Few apoptotic cells were observed in the smooth muscle area of CC after SPC treatment (Figure 5(C)). Parts of the adherens junctions between the SMCs were observed in the SPCtreated group, but they exhibited patterns indicating partial destruction of mitochondria (Figure 5(F)).

3.4. Tracking of Smooth Muscle Progenitor Cells in the Corpus Cavernosum. To determine the potential activity of SPCs, cells were labeled with a long-term tracking dye using the EdU Cell Labeling Kit (Invitrogen) at different time points (6, 24, and 48 hours) and concentrations (10, 50, and $100 \mu \mathrm{M})$. The optimal time point and concentration for EdU 

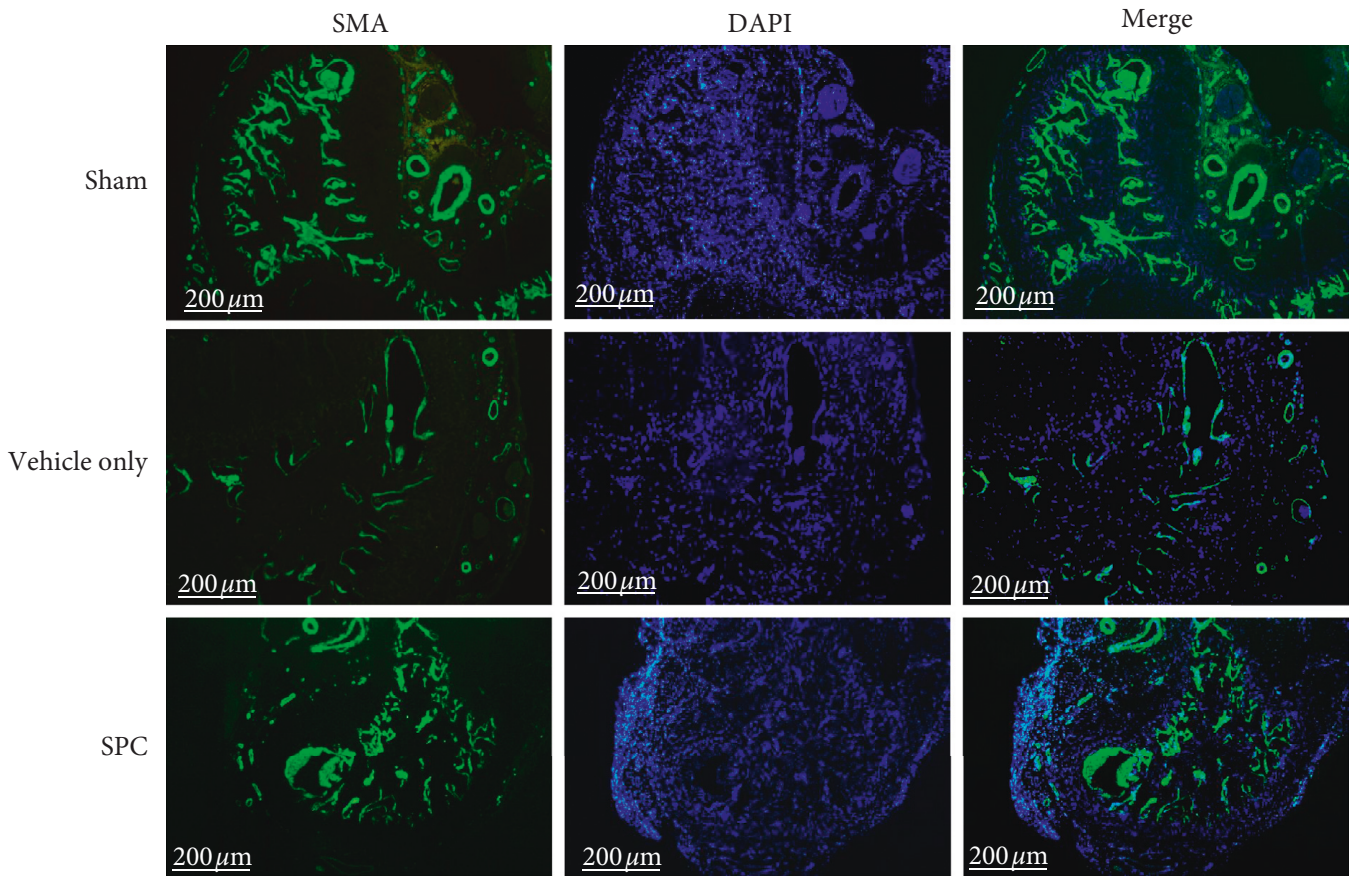

(a)

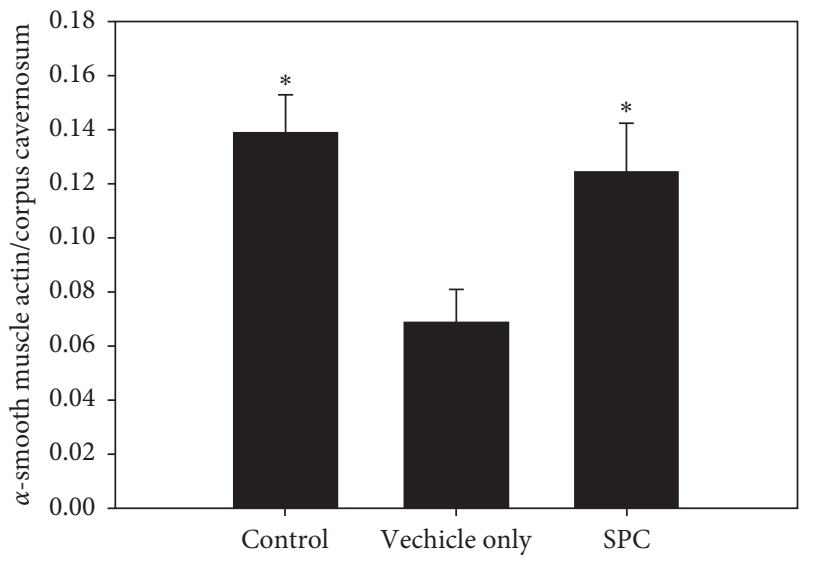

(b)

FIGURE 4: Immunofluorescence staining of $\alpha$-SMA in smooth muscle cell of corpus cavernosum. Histological analyses of the corpus cavernosum (CC) 28 days after injury. (a) Representative fluorescence images of $\alpha$-SMA-positive areas in the rat penile CC (smooth muscle, green; nuclei, blue) (original magnification: 50x). (b) Graph showing the smooth muscle cell (SMC) content in the CC quantified as the $\alpha$-SMA-positive area/CC area. ${ }^{*} p<0.05$ versus the vehicle group.

labeling occurred at 24 hours and at a concentration of $10 \mu \mathrm{M}$, respectively (Figure 6(a)). The locations of EdUpositive (stained red) cells were determined near the vascular and nerve bundle at day 3 and day 7 (Figure 6(b)) and in sinusoid of corpus cavernosum at day 28 after SPC injection in the CC (Figure 6(c)).

\section{Discussion}

In this study, we report the beneficial tissue-protective effects of SPC intracavernosal injection therapy on restoring EF after $\mathrm{CN}$ crush injury. Unfortunately, the analysis of variance in nNOS expression revealed that there were no statistically significant differences between the SPC treatment group and the vehicle only group. However, the SPC treatment group showed significantly greater recovery of EF than the vehicle only group, as indicated by the higher levels of all EF parameters evaluated (including maximum ICP, $\triangle \mathrm{ICP}, \mathrm{AUC}$, and maximum ICP/BP) after treatment with SPCs (for all, $p<0.001$ ). The beneficial tissue-protective effects of SPCs were attributed to an increased maintenance of the structure of the adherens junctions by SMCs due to the inhibition of cell apoptosis, suggesting their involvement in cavernosal tissue remodeling.

The components of penile erection include the cavernous nerve, the endothelium, and corporal smooth muscle. For optimal EF, accurate interactions among these three components are critical [14]. Following the CN crush in animals, 
Sham

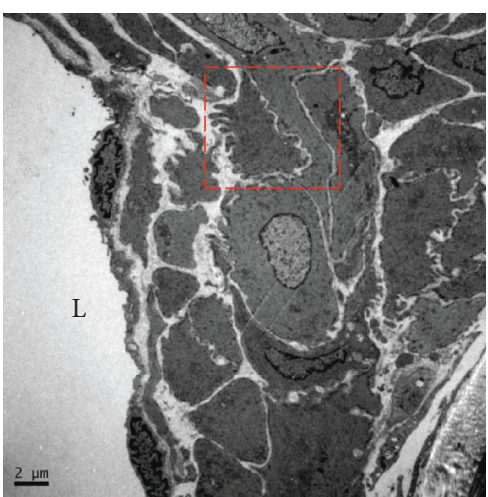

(a)

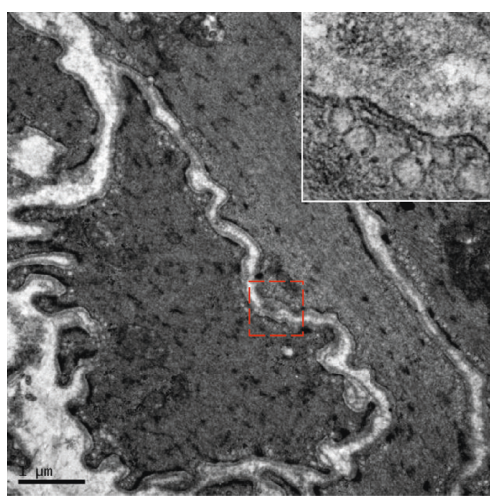

(d)

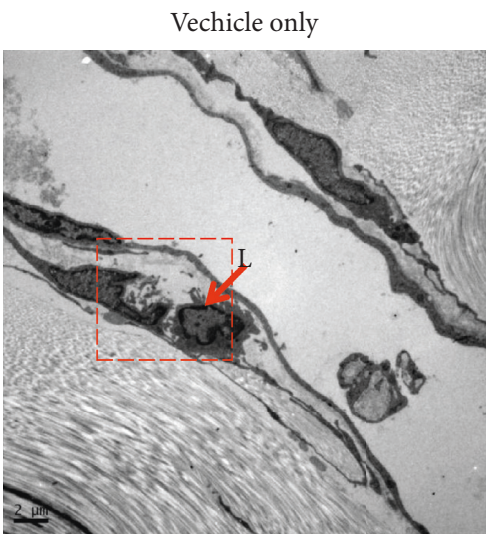

(b)

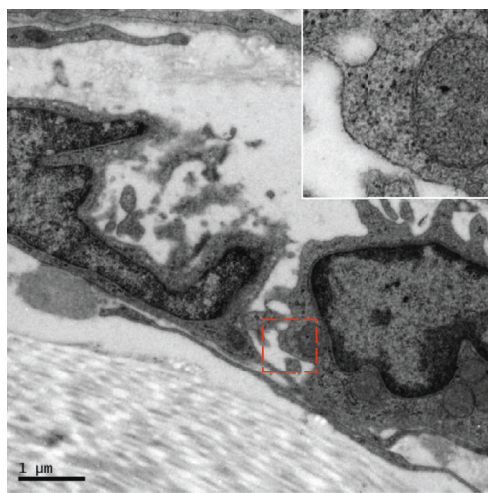

(e)
SPC

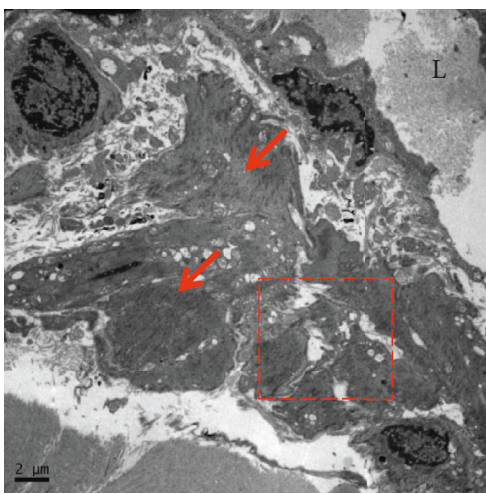

(c)

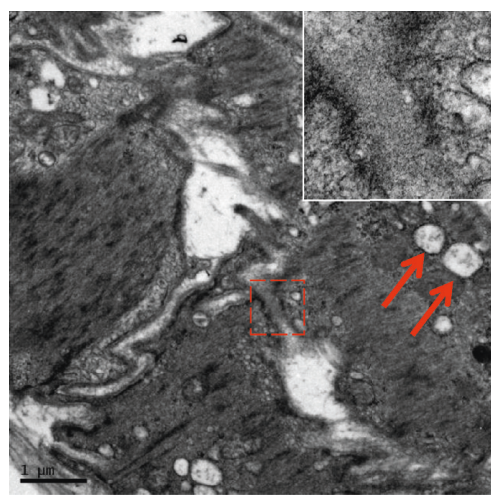

(f)

FIGURE 5: Ultrastructural analysis of the corpus cavernosum (CC) tissue. (a) Transmission electron microscopy (TEM) of the smooth muscle cell (SMC) of the CC near the sinusoid of the sham group. (b) A severe increase in extracellular matrix containing collagen was observed at vehicle only group. (c) Few apoptotic cells are observed in the smooth muscle area of CC after smooth muscle progenitor cells (SPC) treatment. (d) No apparent changes in the adherens junctions of the SMC were observed in the sham group. (e) A few fibroblasts are observed in the smooth muscle apoptotic site and loss of the adherens junctions in the SMC is shown. (f) Part of the adherens junctions between SMCs observed in the SPC group exhibits patterns of partial mitochondrial destruction (red arrow). The upper right square shows higher magnification in all groups.

nNOS expression in the penile nerves rapidly decreases and then begins to increase again over time [18]. The denervation of the penis induces significant apoptosis of corporal SMCs [19]. Corporal smooth muscle fibrosis may cause persistent ED even when other neurological functions recover after operation. In our recent study, we also found the damaged $\mathrm{CN}$ can lead to a partial spontaneous regeneration at 28 days after injury. Defects in the corporal SMCs were irreversible after CN crush injury [10]. Treatment with PDE5 inhibitors increases concentrations of cyclic guanosine monophosphate (cGMP); unfortunately, the composition of the corporal SMC will limit the effects of cGMP. Therefore, the main pathway of PDE5 inhibitor activity is disturbed in conditions of corporal SMC fibrosis. This may explain the low success rate of PDE5 inhibitors in treating post-RP ED. Thus, protecting the corporal SMCs from apoptosis using transplanted progenitor cells may be more important than nerve protection in patients undergoing nerve-sparing RP.

Adherens junctions are protein complexes that exist at cell-cell junctions in various cell types including epithelial, endothelial, and muscle cells, which play an essential role in intercellular tissue connections, mechanotransduction, and smooth muscle contraction [20,21]. In this study, intracavernous injections of SPCs were able to sustain the structure of the adherens junctions of the SMCs, which play a vital role in smooth muscle contraction. In addition, we found that the smooth muscle in the contractile state was abundant around the sinusoid, which suggested that an increased presence of smooth muscle was able to maintain the contractile activity of the SMC intact and resulted in functional improvement. Rats that underwent injection of EdU-labeled SPCs for the purpose of tracking injected SPCs at 4 weeks after $\mathrm{CN}$ injury revealed that EdU-labeled SPCs were recruited to the damaged areas of the CC, implying that the therapeutic effect of SPCs was likely due to their mobilization and differentiation.

Currently, cell-based therapy has become a promising strategy for neuroprotection or tissue-protection after bilateral $\mathrm{CN}$ injury. The stem cell is capable of renewal and repair of tissue due to its capacity for division and differentiation [22]. Intracavernous injection of embryonic stem cells [23] and adult bone marrow-derived stem cells [24] has been reported to improve EF, but these studies have been limited due to clinical applicability and ethical concerns. 
$10 \mu \mathrm{M}$

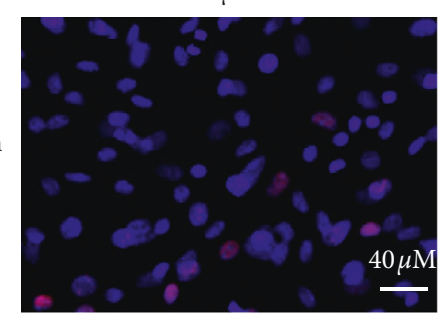

$24 \mathrm{~h}$
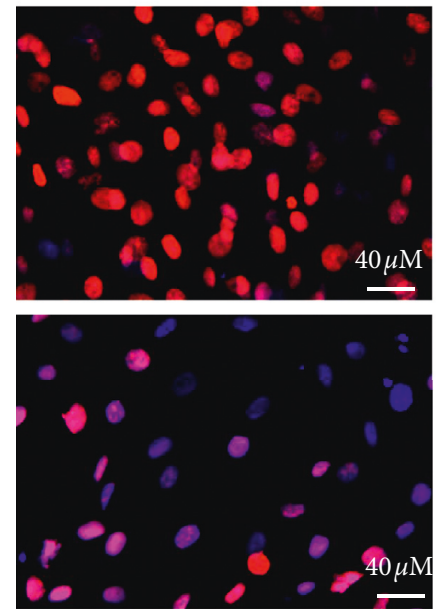

EdU
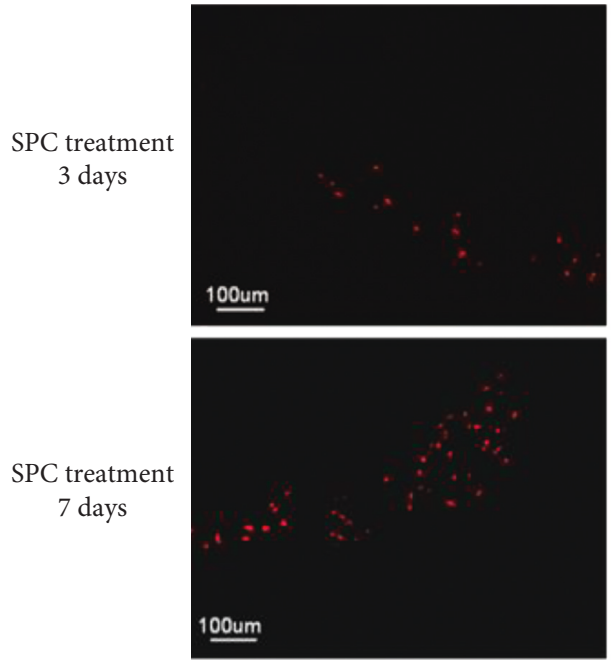

EdU

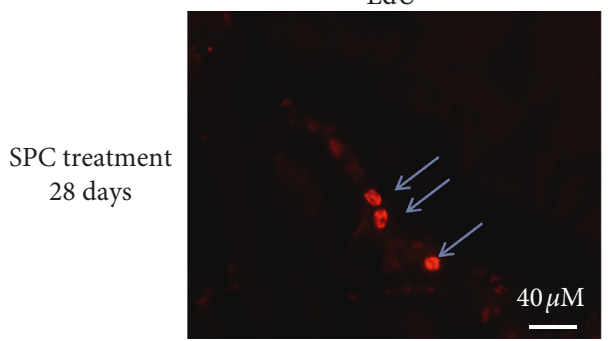

$50 \mu \mathrm{M}$
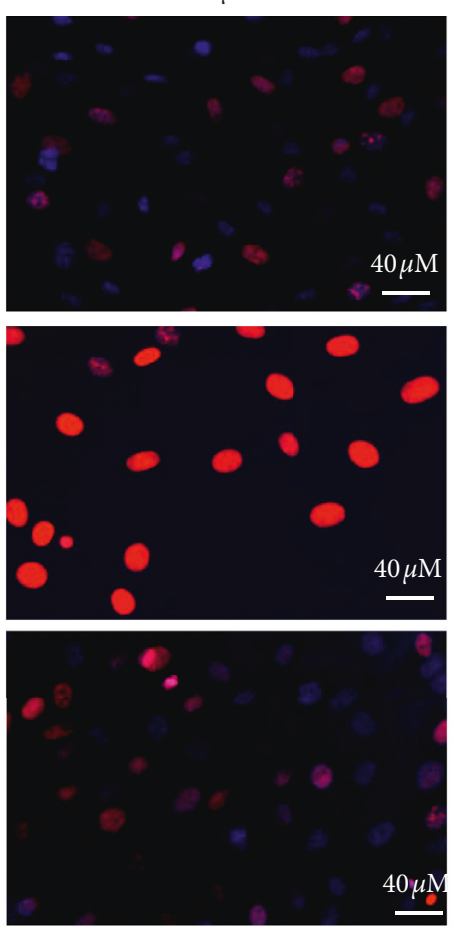

(a)
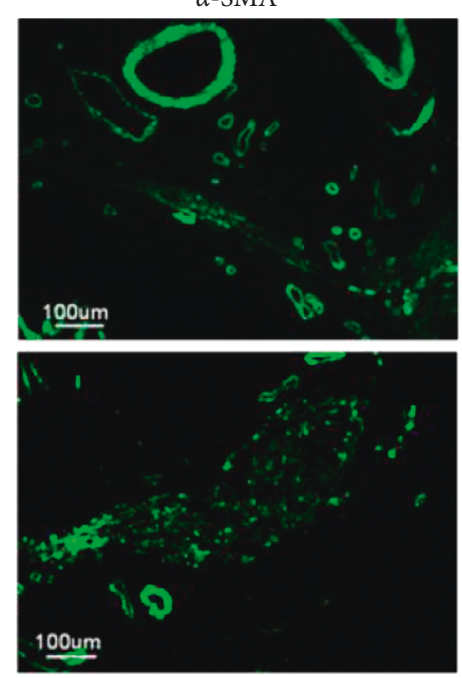

(b)

DAPI

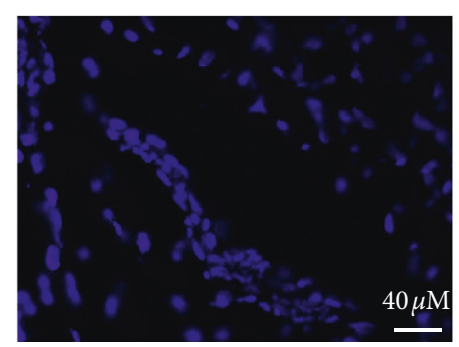

(c)
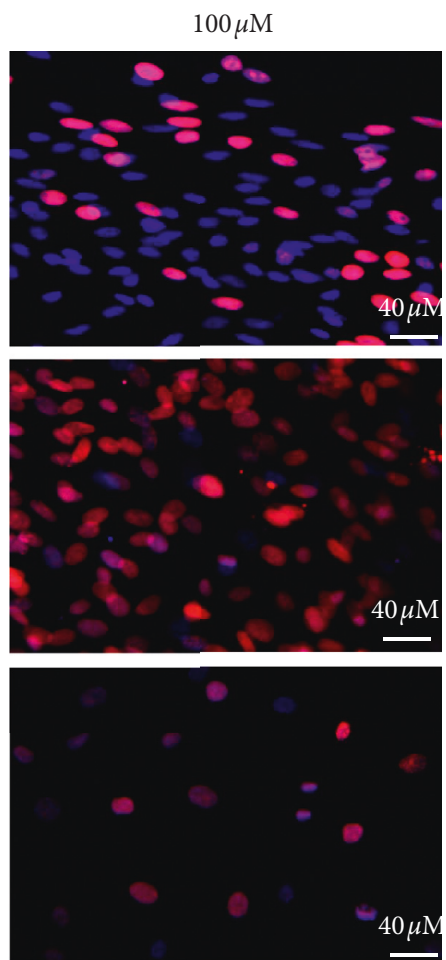

$40 \mu \mathrm{M}$

Merge
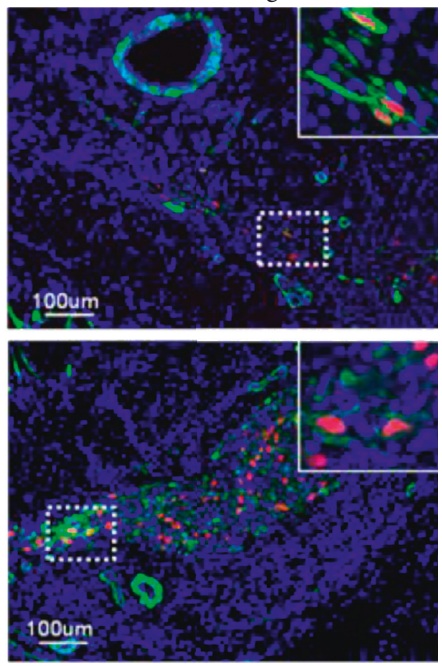

Merge

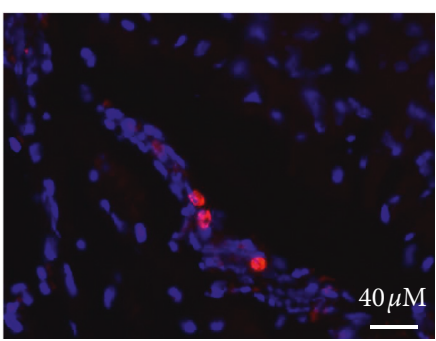

FIGURe 6: EdU labeling of smooth muscle progenitor cells (SPCs). (a) SPCs labeled with EdU at 10, 50, and $100 \mu \mathrm{M}$ and stained with Alexa-594 (red fluorescence) and DAPI (blue fluorescence) (200x magnification). Tracking corpus cavernosum-transplanted SPCs. (b, c) SPCs were labeled with EdU and injected into the corpus cavernosum tissue, which was then harvested at 3, 7, and $28 \mathrm{~d}$ and stained with Alexa-594 (red fluorescence) and DAPI (blue fluorescence). The Alexa-594 and DAPI stained images were digitally merged (scale bar $=40 \mu \mathrm{m}$ and $100 \mu \mathrm{m}$ ). 
Although adipose-derived stem cells (ADSCs) have found application in the regeneration of the $\mathrm{CN}$ in animal models and have been shown to be successful in the recovery of $\mathrm{EF}$ [25], stem cell differentiation does not easily occur during periods of repair [26]. The improvement of EF by ADSCs seems to be attributed to the paracrine action of cytokines rather than to the differentiation of ADSCs [27].

Proangiogenic cell-based therapy using autologous progenitors is a promising strategy for treating ischemic disease [28]. BMPCs, such as EPCs and SPCs, can be derived from peripheral blood and differentiate more easily into vascular cells during arterial remodeling than stem cells. EPCs are capable of circulating, proliferating, and differentiating into mature endothelial cells [13]. EPCs are required for endothelial repair and can participate in blood vessel formation [29]. The cavernous endothelium plays a crucial role in regulating the tone of the underlying smooth muscle and physiological penile erection [30]. We also reported previously that EPC treatment restored EF in a rat model of bilateral CN injury through the recruitment of EPCs toward the dorsal artery and preservation of SMCs in the CC. These findings corroborate the therapeutic potential of EPCs for treating ED in humans [14]. SPC-released angiopoietin-1 can facilitate stabilization of endothelial cell networks and may be more suitable to tightly orchestrate the complex process of neovascularization [16]. In addition, administration of SPCs can thicken the vascular intima and has demonstrated some therapeutic benefit for cardiovascular disease in animal models [31]. A tight cooperation between endothelial cells and SMCs is important to regulate vessel maturation and stability. Foubert et al. stated that SPCs may enhance the efficiency of treatment with EPCs [17]. More importantly, the proliferation ability of SPCs in vitro is higher than that of EPCs. Our study is the first report describing the use of SPCs to treat ED after $\mathrm{CN}$ injury and further elucidates the clinical application of SPCs in humans.

\section{Conclusions}

Treatment with SPCs restored EF in a rat model of bilateral $\mathrm{CN}$ crush injury through the recruitment of SPCs toward the sinuses in the corpora cavernosa, which sustained the structure of the adherens junctions of the SMC through a reduction of cellular apoptosis and increased stability of the corporal vessel. These findings provide support for the therapeutic potential of SPCs in treating ED in humans.

\section{Abbreviations}

ED: Erectile dysfunction

CC: $\quad$ Corpus cavernosum

SMC: $\quad$ Smooth muscle cell

SPCs: Smooth muscle progenitor cells

$\mathrm{CN}$ : Cavernous nerve

EPCs: Endothelial progenitor cells

BMPCs: Bone marrow-derived progenitor cells

ICP: Intracavernosal pressure

BP: $\quad$ Blood pressure

BMMCs: Bone marrow mononuclear cells
ADSCs: Adipose-derived stem cells.

\section{Data Availability}

The data used to support the findings of this study are available from the corresponding author upon request.

\section{Ethical Approval}

The protocol of this study was reviewed and approved by the $\mathrm{Fu}$ Jen Catholic University Animal Care and Use Committee (IACUC approval no.: A10425).

\section{Disclosure}

An earlier version of this study was presented as an abstract in the 2018 33rd Joint Annual Conference of Biomedical Science.

\section{Conflicts of Interest}

The authors declare that they have no conflicts of interest.

\section{Authors' Contributions}

Yi-No Wu contributed to the conception and design, collection and/or assembly of data, data analysis and interpretation, and manuscript writing. Kuo-Chiang Chen approved the final manuscript. Chun-Hou Liao was involved in conception and design, data analysis and interpretation, and final approval of the manuscript. Chien-Liang Liu performed data analysis and interpretation. Han-Sun Chiang was responsible for conception and design and final approval of the manuscript.

\section{Acknowledgments}

The authors would like to thank Mr. Yen-Sheng Wu of Tzong Jwo Jang's electron microscope laboratory and Fu Jen Catholic University for technical assistance. This work was supported by the National Science Council (MOST 1052314-B-030-010), the Chi Mei Hospital and Cardinal Tien Hospital and $\mathrm{Fu}$ Jen Catholic University Education and Development Cooperation Project (CM-104CM-FJU-01) (CTH-103-1-2B11) (107-CTH-FJU-02), and the Cathay General Hospital and Fu Jen Catholic University Education and Development Cooperation Project (107-CGH-FJU-09).

\section{References}

[1] R. Wang, "Penile rehabilitation after radical prostatectomy: where do we stand and where are we going?," The Journal of Sexual Medicine, vol. 4, no. 4, pp. 1085-1097, 2007.

[2] A. L. Burnett, "Rationale for cavernous nerve restorative therapy to preserve erectile function after radical prostatectomy," Urology, vol. 61, no. 3, pp. 491-497, 2003.

[3] J. H. Kim and S. W. Lee, "Current status of penile rehabilitation after radical prostatectomy," Korean Journal of Urology, vol. 56, no. 2, pp. 99-108, 2015. 
[4] J. Mulcahy, "Surgical management of penile prosthesis complications," International Journal of Impotence Research, vol. 12, no. S4, pp. S108-S111, 2000.

[5] W. Jin and Y. R. Huang, "Treatment of erectile dysfunction after radical retropubic prostatectomy with PDE5 inhibitor," Zhonghua Nan Ke Xue, vol. 11, pp. 708-712, 2005.

[6] F. Montorsi, G. Brock, J.-U. Stolzenburg et al., "Effects of tadalafil treatment on erectile function recovery following bilateral nerve-sparing radical prostatectomy: a randomised placebo-controlled study (REACTT)," European Urology, vol. 65, no. 3, pp. 587-596, 2014.

[7] Y.-N. Wu, C.-C. Wu, M.-T. Sheu, K.-C. Chen, H.-O. Ho, and H.-S. Chiang, "Optimization of platelet-rich plasma and its effects on the recovery of erectile function after bilateral cavernous nerve injury in a rat model," Journal of Tissue Engineering and Regenerative Medicine, vol. 10, no. 10, pp. E294-E304, 2016.

[8] C. C. Wu, Y. N. Wu, H. O. Ho, K. C. Chen, M. T. Sheu, and H. S. Chiang, "The neuroprotective effect of platelet-rich plasma on erectile function in bilateral cavernous nerve injury rat model," The Journal of Sexual Medicine, vol. 9, no. 11, pp. 2838-2848, 2012.

[9] C. H. Liao, Y. N. Wu, B. H. Chen, Y. H. Lin, H. O. Ho, and H. S. Chiang, "Neuroprotective effect of docosahexaenoic acid nanoemulsion on erectile function in a rat model of bilateral cavernous nerve injury," Scientific Reports, vol. 6, no. 1, 2016.

[10] Y. N. Wu, K. C. Chen, C. H. Liao, and H. S. Chiang, "Spontaneous regeneration of nerve fiber and irreversibility of corporal smooth muscle fibrosis after cavernous nerve crush injury: evidence from serial transmission electron microscopy and intracavernous pressure," Urology, vol. 118, pp. 98-106, 2017.

[11] S. Leungwattanakij, T. J. Bivalacqua, M. F. Usta et al., "Cavernous neurotomy causes hypoxia and fibrosis in rat corpus cavernosum," Journal of Andrology, vol. 24, no. 2, pp. 239-245, 2003.

[12] D. You, M. J. Jang, B. H. Kim et al., "Comparative study of autologous stromal vascular fraction and adipose-derived stem cells for erectile function recovery in a rat model of cavernous nerve injury," Stem Cells Translational Medicine, vol. 4, no. 4, pp. 351-358, 2015.

[13] T. Asahara, T. Murohara, A. Sullivan et al., "Isolation of putative progenitor endothelial cells for angiogenesis," Science, vol. 275, no. 5302, pp. 964-966, 1997.

[14] C.-H. Liao, Y.-N. Wu, Y.-H. Lin, R.-F. Syu Huang, S.-P. Liu, and H.-S. Chiang, "Restoration of erectile function with intracavernous injections of endothelial progenitor cells after bilateral cavernous nerve injury in rats," Andrology, vol. 3, no. 5, pp. 924-932, 2015.

[15] T. Merkulova-Rainon, D. Broqueres-You, N. Kubis, J.-S. Silvestre, and B. I. Levy, "Towards the therapeutic use of vascular smooth muscle progenitor cells," Cardiovascular Research, vol. 95, no. 2, pp. 205-214, 2012.

[16] P. Foubert, G. Matrone, B. Souttou et al., "Coadministration of endothelial and smooth muscle progenitor cells enhances the efficiency of proangiogenic cell-based therapy," Circulation Research, vol. 103, no. 7, pp. 751-760, 2008.

[17] P. Foubert, C. Squiban, V. Holler et al., "Strategies to enhance the efficiency of endothelial progenitor cell therapy by ephrin B2 pretreatment and coadministration with smooth muscle progenitor cells on vascular function during the woundhealing process in irradiated or nonirradiated condition," Cell Transplantation, vol. 24, no. 7, pp. 1343-1361, 2015.
[18] T. M. Fandel, M. Albersen, G. Lin et al., "Recruitment of intracavernously injected adipose-derived stem cells to the major pelvic ganglion improves erectile function in a rat model of cavernous nerve injury," European Urology, vol. 61, no. 1, pp. 201-210, 2012.

[19] H. M. User, J. H. Hairston, D. J. Zelner, K. E. McKenna, and K. T. McVary, "Penile weight and cell subtype specific changes in a post-radical prostatectomy model of erectile dysfunction," Journal of Urology, vol. 169, no. 3, pp. 1175-1179, 2003.

[20] T. Wang, R. Wang, R. A. Cleary, O. J. Gannon, and D. D. Tang, "Recruitment of $\beta$-catenin to $N$-cadherin is necessary for smooth muscle contraction," Journal of Biological Chemistry, vol. 290, no. 14, pp. 8913-8924, 2015.

[21] A. M. Belkin, I. V. Klimanskaya, M. E. Lukashev, K. Lilley, D. R. Critchley, and V. E. Koteliansky, "A novel phosphoglucomutase-related protein is concentrated in adherens junctions of muscle and nonmuscle cells," Journal of Cell Science, vol. 107, pp. 159-173, 1994.

[22] A. B. Reed-Maldonado and T. F. Lue, "The current status of stem-cell therapy in erectile dysfunction: a review," The World Journal of Men's Health, vol. 34, no. 3, pp. 155-164, 2016.

[23] D. Bochinski, G. T. Lin, L. Nunes et al., "The effect of neural embryonic stem cell therapy in a rat model of cavernosal nerve injury," BJU International, vol. 94, no. 6, pp. 904-909, 2004.

[24] P. A. Fall, M. Izikki, L. Tu et al., "Apoptosis and effects of intracavernous bone marrow cell injection in a rat model of postprostatectomy erectile dysfunction," European Urology, vol. 56, no. 4, pp. 716-726, 2009.

[25] H. Valentine, Y. Chen, H. Guo et al., "Neuroimmunophilin ligands protect cavernous nerves after crush injury in the rat: new experimental paradigms," European Urology, vol. 51, no. 6, pp. 1724-1731, 2007.

[26] G. Lin, L. Banie, H. Ning, A. J. Bella, C. S. Lin, and T. F. Lue, "Potential of adipose-derived stem cells for treatment of erectile dysfunction," The Journal of Sexual Medicine, vol. 6, pp. 320-327, 2009.

[27] H. Zhang, R. Yang, Z. Wang, G. Lin, T. F. Lue, and C. S. Lin, "Adipose tissue-derived stem cells secrete CXCL5 cytokine with neurotrophic effects on cavernous nerve regeneration," The Journal of Sexual Medicine, vol. 8, no. 2, pp. 437-446, 2011.

[28] H. J. Joo, H.-R. Seo, H. E. Jeong et al., "Smooth muscle progenitor cells from peripheral blood promote the neovascularization of endothelial colony-forming cells," Biochemical and Biophysical Research Communications, vol. 449, no. 4, pp. 405-411, 2014.

[29] B. Doyle, P. Metharom, and N. M. Caplice, "Endothelial progenitor cells," Endothelium, vol. 13, no. 6, pp. 403-410, 2006.

[30] T. J. Bivalacqua, M. F. Usta, H. C. Champion, P. J. Kadowitz, and W. J. G. Hellstrom, "Endothelial dysfunction in erectile dysfunction: role of the endothelium in erectile physiology and disease," Journal of Andrology, vol. 24, no. S6, pp. S17S37, 2003.

[31] S. Akhtar, F. Gremse, F. Kiessling, C. Weber, and A. Schober, "CXCL12 promotes the stabilization of atherosclerotic lesions mediated by smooth muscle progenitor cells in apoe-deficient mice," Arteriosclerosis, Thrombosis, and Vascular Biology, vol. 33, no. 4, pp. 679-686, 2013. 


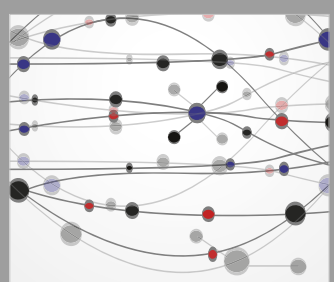

The Scientific World Journal
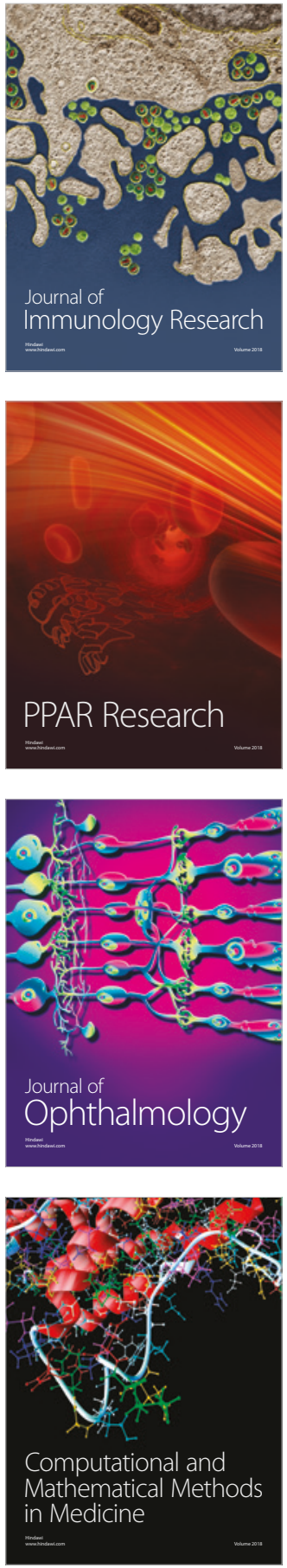

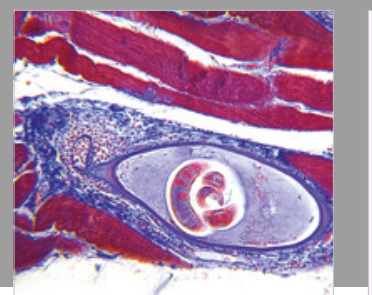

Gastroenterology Research and Practice

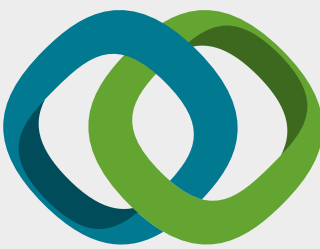

\section{Hindawi}

Submit your manuscripts at

www.hindawi.com
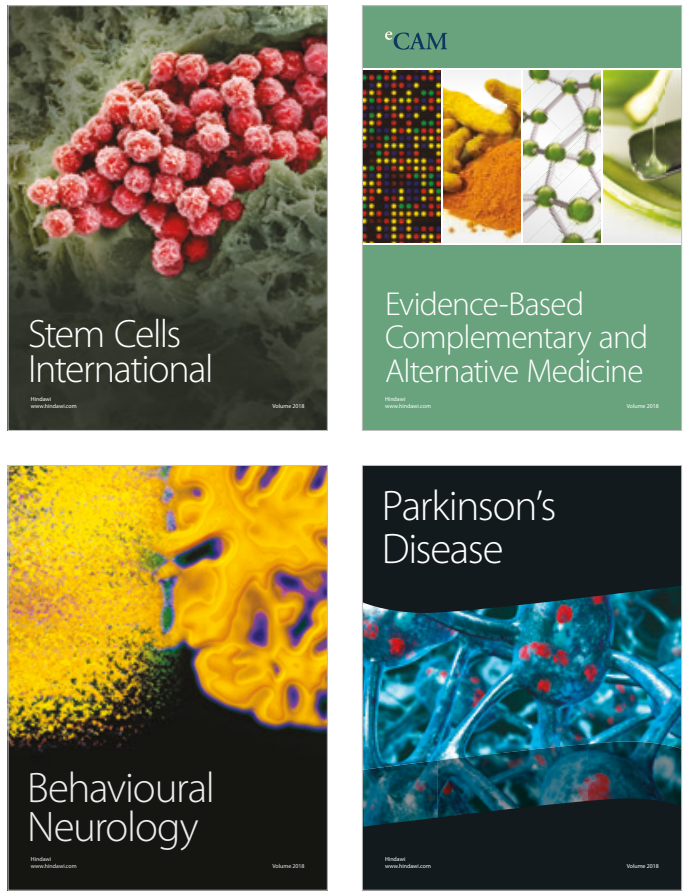

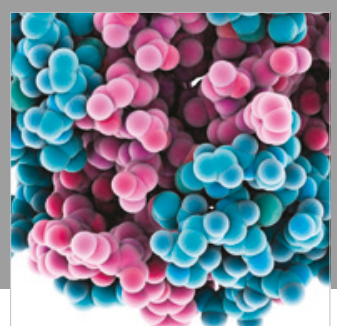

ournal of

Diabetes Research

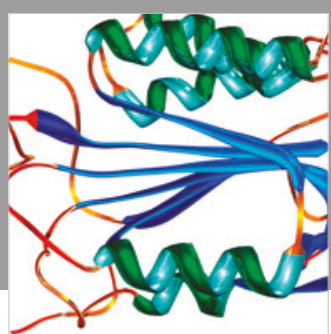

Disease Markers
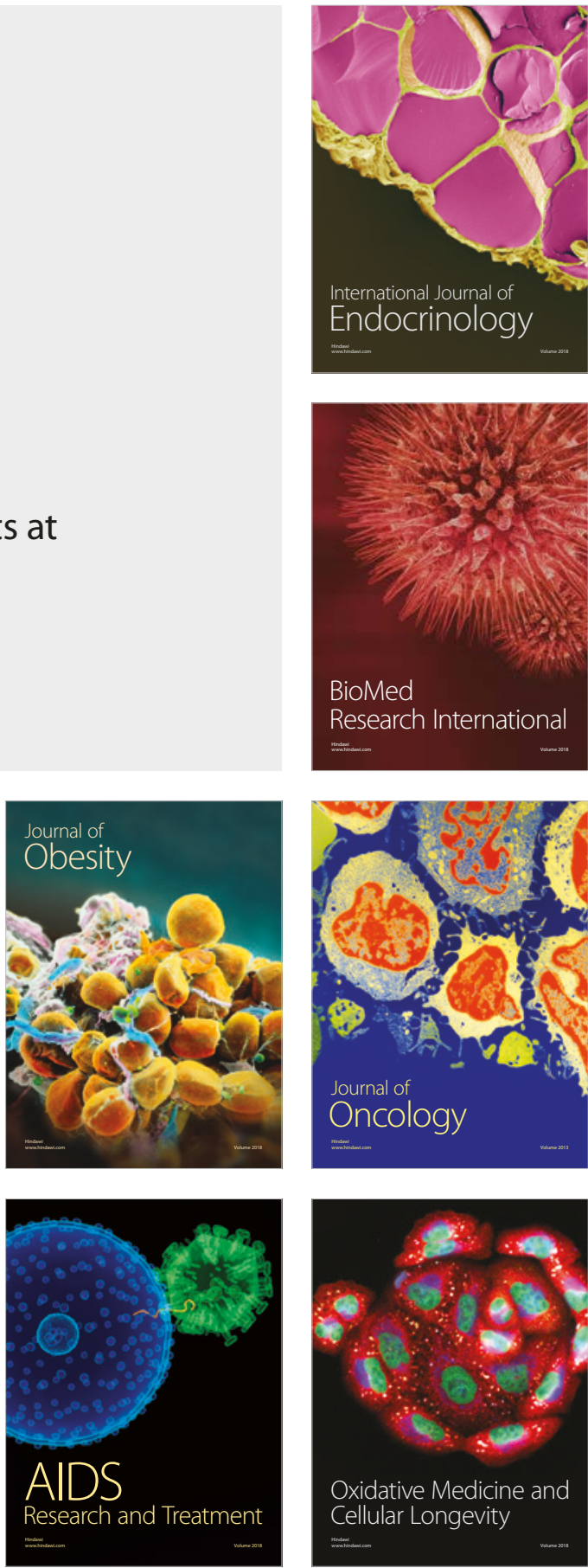